\title{
PROPOSTA E VALIDAÇÃO DE INDICADORES HIDROAMBIENTAIS PARA BACIAS HIDRO- GRÁFICAS: ESTUDO DE CASO NA SUB-BACIA DO ALTO CURSO DO RIO PARAÍBA, PB.
}

\author{
Proposal and Validation of Indicators for Hydro-environmental Watershed: \\ A Case Study in the Sub-Basin of the Upper Course of the River Paraíba, PB.
}

José Ribamar Marques de Carvalho

Doutorando em Recursos Naturais - PPGRN/UFCG

Professor da Unidade Acadêmica de Ciências Contábeis - CCJS/UFCG

Sousa/ PB - Brasil.

profribamar@gmail.com

Wilson Fadlo Curi

Doutor e Professor do Programa de Pós-Graduação em Recursos Naturais - PPGRN/UFCG

Centro de Tecnologia e Recursos Naturais - CTRN/UFCG

Campina Grande/ PB - Brasil wfcuri@yahoo.com.br

Enyedja Kerlly Martins de Araújo Carvalho Mestranda em Recursos Naturais - PPGRN/UFCG

Campina Grande/ PB - Brasil.

enyedjakm@gmail.com

Rosires Catão Curi

Doutora e Professora do Programa de Pós-Graduação em Recursos Naturais - PPGRN/UFCG

Centro de Tecnologia e Recursos Naturais - CTRN/UFCG

Campina Grande/ PB - Brasil. rosirescuri@yahoo.com.br

Artigo recebido para publicação em 11/05/2011 e aceito para publicação em 08/07/2011.

RESUMO : Dentre as formas de subsidiar uma gestão dos recursos hídricos, capaz de contribuir para a busca do desenvolvimento sustentável, têm-se as ferramentas adequadas de medir o desempenho dos sistemas hídricos e ambientais. Nesse contexto, e em meio a uma diversidade de sistemas de indicadores de sustentabilidade hidroambientais existentes, foi elaborada essa proposta, composta por 51 indicadores, com o objetivo de verificar o nível de sustentabilidade hidroambiental dos municípios localizados na sub-bacia hidrográfica do Alto Curso do Rio Paraíba, PB. A metodologia utilizada consistiu em uma pesquisa exploratória e documental, na qual fez o uso da estatística descritiva. Os resultados obtidos apontam que os municípios com melhores desempenhos hidroambiental foram: Boqueirão, Monteiro, Sumé, Ouro Velho e Serra Branca. No outro ponto os municípios com piores desempenhos foram: Prata, Coxixola, Congo, São João do Cariri, Camalaú, São Domingos do Cariri, Zabelê, Cabaceiras, Barra de São Miguel, São Sebastião do Umbuzeiro, Amparo e São João do Tigre. Esses resultados demonstram a necessidade de definir programas de gestão hídrica capazes de reverter esse cenário de instabilidade, através de ações mais responsáveis por parte das entidades reguladoras da gestão de águas e demais entidades de classe no estado paraibano e em especial na sub-bacia estudada. 
Palavras-chave: Indicadores hidroambientais. Sustentabilidade hídrica. Bacia hidrográfica.

ABSTRACT: $\quad$ Among the ways to subsidize a water resources management, capable of contribute to the pursuit of sustainable development, have the tools appropriate to measure the performance of water systems and environmental issues. In this context, and amid a variety of measurement systems hydro-environmental existing sustainability, this proposal was drafted, composed of 51 indicators, with the objective of verifying the level of hydro-environmental sustainability of municipalities in the sub-basin of the Upper Course of the River Paraíba, PB. The methodology consisted of an exploratory research and documentary, in which he made use of descriptive statistics. The results suggest that municipalities with hydro-environmental best performances were: Boqueirão, Monteiro, Sumé, Ouro Velho and Serra Branca. At another point the municipalities with the worst performance were: Prata, Coxixola, Congo, São João do Cariri, Camalaú, São Domingos do Cariri, Zabelê, Cabaceiras, Barra de São Miguel, São Sebastião do Umbuzeiro, Amparo e São João do Tigre. These results demonstrate the need for defining water management programs that can reverse this scenario instability through more responsible actions by entities regulators of water management and other professional associations in the state Paraíba and especially in sub-basin studied.

Keywords: Indicators hydro-environmental. Water sustainability. Watershed.

\section{INTRODUÇÃO}

A problemática que permeia o meio ambiente fruto das pressões humanas para com a natureza fez com que no atual contexto surgisse um tema de grande relevância - o desenvolvimento sustentável, que passou a ser um dos temas mais debatidos e estudados nos meios acadêmicos e científicos, governamentais.

Seu conceito tornou-se público em 1972, após as reuniões da ONU (Organização das Nações Unidas) sobre meio ambiente, realizadas em Estocolmo. Entretanto, somente em 1987 a Comissão Mundial para o Meio Ambiente e Desenvolvimento da ONU (WCED) emitiu a primeira definição concisa onde destacava que o desenvolvimento sustentável é aquele que satisfaz as necessidades das gerações presentes sem comprometer a capacidade das gerações futuras de satisfazerem as suas próprias necessidades. (DANIEL, 2000)

Muito embora esse conceito apresente algumas fragilidades, proporcionou estimular a integração e a equidade entre várias gerações, tendo sido rapidamente compreendido e adotado pela comunidade em geral. (DANIEL, 2000). Todavia, esse termo passou a gerar polêmicas em função dos poucos avanços, em termos de consenso, obtidos quando se trata desta questão [...], principalmente se for considerado como um pano de fundo de toda esta discussão que tomou dimensões globais. (MOURA, 2002)

Os debates sobre estratégias para conciliar o crescimento econômico com as necessidades sociais e ambientais assumiu, nos campos político e científico, uma dimensão global. O livro Primavera Silenciosa de Rachel Carson, publicado em 1962, o Relatório de Brundtland de 1987 e a Conferência ECO-92, no Rio de Janeiro, podem ser considerados emblemáticos na mudança do enfoque de desenvolvimento no sentido de possibilitar a satisfação das necessidades do presente sem comprometer as possibilidades de sobrevivência das gerações futuras. (MOURA, 2002).

Essa preocupação em rever o conceito de desenvolvimento mostrou que a noção de progresso associada à ideia de crescimento vivenciada durante a Revolução Industrial do Século XX, necessitava ser examinada em função das crises econômicas, ambientais, sociais e político-institucionais, de modo que pudesse ser substituída por uma interação mais arrojada em relação à sociedade-natureza e em especial dos recursos hídricos.

No Brasil, país de dimensões continentais, estão localizadas algumas das maiores bacias hidro- 
Proposta e validação de indicadores hidroambientais para bacias hidrográficas: estudo de caso na sub-bacia do alto curso do Rio Paraíba, PB. José Ribamar Marques de Carvalho, Wilson Fadlo Curi, Enyedja Kerlly Martins de Araújo Carvalho, Rosires Catão Curi

gráficas do mundo e, não por acaso, cerca de $90 \%$ de energia elétrica produzida tem origem hidráulica; essa enorme disponibilidade levou o povo brasileiro a encobrir o desperdício e o uso predatório com a capa da abundância e a justificar essa conduta introjetando a ideia de que a água era um bem invulnerável, ao qual todos podiam e poderão ter sempre acesso ilimitado. (MARANHÃO, 2007. p. 16)

Observa-se que um dos maiores desafios enfrentados na quantificação ou qualificação da sustentabilidade consiste na elaboração de metodologias adequadas que permitam avaliar a sustentabilidade de realidades locais, regionais ou nacionais, posto existirem diferentes características e peculiaridades inerentes aos aspectos sociais, econômicos, ambientais, culturais e institucionais.

Vários parâmetros e variáveis têm sido apontados na literatura para inferir o desempenho de sistemas hídricos e ambientais - são os chamados 'indicadores'. A dificuldade, na verdade, não parece estar em apontar indicadores, mas em agregá-los em um único parâmetro - o 'índice' - capaz de traduzir numericamente uma situação e apontar, ao tomador de decisão, o sentido da sustentabilidade da região. (VIEIRA; STUDART, 2009, p. 125)

Em meio a uma diversidade de Sistemas de Indicadores de Sustentabilidade Hidroambientais existentes, foi elaborada essa proposta especificamente a partir dos estudos de Guimarães (2008), Martins e Cândido (2008), Vieira e Studart (2009), Magalhães Júnior (2010) com o objetivo de verificar o nível de sustentabilidade da sub-bacia hidrográfica do Alto Piranhas, PB, ou seja, verificar quais municípios se encontram em situação mais insustentável/sustentável em relação à sustentabilidade hídrica.

O motivo que justifica a execução desse estudo concentra-se no fato de que atualmente as pressões sobre os corpos hídricos têm aumentado significativamente e em especial na região do semiárido nordestino onde a média pluviométrica é baixa e necessita de um maior planejamento e consequentemente gestão dos recursos hídricos e ainda pelo fato de não existir trabalhos desenvolvidos neste contexto geográfico.

\section{REFERENCIAL TEÓRICO}

\section{Importância e Peculiaridades dos Indicadores de Sustentabilidade}

O homem é o grande agente transformador do ambiente natural e vem, pelo menos há doze milênios, promovendo essas adaptações nas mais variadas localizações climáticas, geográficas e topográficas. O ambiente urbano é, portanto, o resultado de aglomerações localizadas em ambientes naturais transformados, e que para a sua sobrevivência e desenvolvimento necessitam dos recursos do ambiente natural. (PHILIPPI JR. et al. 2004)

Assim, a maneira de gerir a utilização desses recursos é o fator que pode acentuar ou minimizar os impactos. Esse processo de acordo com Philipp Jr. e outros (2004) fundamenta-se em três variáveis: a diversidade dos recursos extraídos do ambiente natural, a velocidade de extração desses recursos, que permitem ou não a sua reposição, e a forma de disposição e tratamento de seus resíduos e efluentes. A somatória dessas três variáveis e a maneira de geri-las definem o grau de impacto do ambiente urbano sobre o ambiente natural, nos quais poderão e dependendo dos níveis associados pode sucumbir a sustentabilidade ambiental.

O maior desafio, quando se trata de discutir a questão da sustentabilidade, é o de compatibilizar o crescimento econômico com a preservação ambiental e justiça social. Para isso, o gerenciamento do conhecimento no uso dos indicadores de sustentabilidade pode ser um instrumento adequado para tais evidências, principalmente porque nestes sistemas de indicadores estão contidas todas as informações pertinentes à situação econômica, social, e ambiental de um espaço geográfico em um determinado período. (LIRA, 2008. p. 72)

De acordo com Kronemberger e outros (2008) o Índice de Sustentabilidade é uma forma de sintetizar, matematicamente, uma série de informações quantitativas e semi-quantitativas, associadas à sustentabilidade do desenvolvimento. Cada índice, ao final, produz um valor numérico, resultado de operações matemáticas com as informações que utiliza, e, quando comparado a uma escala padrão, avalia a sustentabilidade.

Os indicadores de sustentabilidade aparecem como ferramentas capazes de subsidiar o monito- 
Proposta e validação de indicadores hidroambientais para bacias hidrográficas: estudo de caso na sub-bacia do alto curso do Rio Paraíba, PB. José Ribamar Marques de Carvalho, Wilson Fadlo Curi, Enyedja Kerlly Martins de Araújo Carvalho, Rosires Catão Curi

ramento da operacionalização do desenvolvimento sustentável, tendo como função principal a revelação de informações sobre o estado das diversas dimensões (ambientais, econômicas, socioeconômicas, culturais, institucionais etc) que compõem o desenvolvimento sustentável do sistema na sociedade.

Os indicadores de sustentabilidade são utilizados como ferramenta padrão em diversos estudos nacionais e internacionais, facilitando a compreensão das informações sobre fenômenos complexos, e atua como base para análise do desenvolvimento que abrange diversas dimensões (nelas incluídas fatores econômicos, sociais, culturais, geográficos e ambientais), uma vez que permite verificar os impactos das ações humanas no ecossistema. (SILVA, A. M.; CORREIA, A. M. M.; CÂNDIDO, G. A. 2010, p. 242)

As definições mais comuns de indicadores e a terminologia associada a essa área são particularmente confusas, tanto em relação à definição de indicadores quanto a outros conceitos associados como: índice, meta e padrão. O objetivo dos indicadores é agregar e quantificar informações de um modo que sua significância fique mais aparente, simplificando informações sobre fenômenos complexos tentando melhorar com isso o processo de comunicação. Percebe-se que os indicadores podem ser quantitativos ou qualitativos, existindo autores que definem que os mais adequados para a avaliação de experiências de desenvolvimento sustentável deveriam ser mais qualitativos, em função das limitações explícitas e implícitas que existem em relação a indicadores simplesmente numéricos, muito embora, em alguns casos, avaliações qualitativas podem ser transformadas numa notação quantitativa. (VAN BELLEN, 2006)

$\mathrm{O}$ que se percebe no discurso exposto é que a utilização de indicadores é uma maneira intuitiva de monitorar complexos sistemas, que a sociedade considera importantes e que devem ser controlados, capazes de evidenciar elementos importantes da maneira como a sociedade entende seu mundo, toma duas decisões e planeja a sua ação. Os valores, e logicamente os indicadores, estão inseridos dentro de culturas específicas, muito embora existam armadilhas na sua utilização e ainda que estejam inseridos dentro de culturas específicas, realçando o que está acontecendo em determinada sociedade.

Fica evidente que muito embora existam várias definições acerca da terminologia utilizada, ainda permanece a falta de consenso entre os autores, emergindo desta forma a necessidade de desenvolver uma definição mais rigorosa e unificada de indicador no que se refere à temática ambiental, visto que, a grande maioria dos sistemas de indicadores existentes e utilizados foi desenvolvida por razões específicas, sejam elas ambientais, econômicas, de saúde e sociais e não podem ser considerados indicadores de sustentabilidade em si, mesmo dispondo de um potencial representativo dentro do contexto do desenvolvimento sustentável conforme defende Van Bellen (2006).

Alguns cuidados chaves devem ser tomados quando da escolha de indicadores, uma vez que, diferentes tipos de indicadores podem em determinadas situações ser relevantes em diferentes escalas, e, também podem perder o seu sentido quando utilizados sem o devido cuidado em escalas não apropriadas. Um outro aspecto destacado na discussão dos indicadores relacionados ao desenvolvimento sustentável, é destacado por Van Bellen (2006), que consiste na dimensão do tempo podendo ser escalares (número simples gerado da agregação de dois ou mais valores) e vetoriais (geração de uma variável com magnitude e direção de característica bidirecional - tendência de futuro com viés holístico), além dos valores existentes na sua escolha, explícitos (tomados conscientemente e que compreendem uma parte fundamental no processo de criação de indicadores) e os implícitos (decorrem de aspectos que não são facilmente observáveis e que são, na sua maioria, inconscientes e relacionados a características pessoais e de uma determinada sociedade).

Exaustivamente o autor elenca outras características e ou requisitos como ponderação, contexto geográfico, temporal, econômico, local, cultura, compreensibilidade, mensuráveis, ter disponibilidade de dados, metodologia para coleta e processamento de dados, viabilidade financeira, humana e técnica e ainda aceitação política.

\section{PROCEDIMENTOS METODOLÓGICOS}

A metodologia utilizada neste estudo consistiu em uma pesquisa documental e exploratória, na qual fez o uso da estatística descritiva. Foram escolhidos nesta primeira fase alguns indicadores hidroambien- 
Proposta e validação de indicadores hidroambientais para bacias hidrográficas: estudo de caso na sub-bacia do alto curso do Rio Paraíba, PB. José Ribamar Marques de Carvalho, Wilson Fadlo Curi, Enyedja Kerlly Martins de Araújo Carvalho, Rosires Catão Curi

tais de maneira que fosse possível identificar aspectos da sustentabilidade hídrica. Os estudos que embasaram a escolha dos indicadores foram os trabalhos de Guimarães (2008), Martins e Cândido (2008), Vieira e Studart (2009), Magalhães Júnior (2010).

Para este trabalho, foram buscados dados cujas diferenças temporais fossem as menores possíveis e ainda que levassem em consideração a disponibilidade dos mesmos e o critério de escolha dos autores. Observou-se que as variáveis estudadas apresentam diferentes unidades de medida, fazendo com que fossem utilizadas unidades de agregação a partir das relações positivas e negativas de cada uma delas. O procedimento procurou ajustar os valores observados das variáveis a escalas cujo valor mínimo é 0 (zero) e valor máximo é igual a 1 (um), criando condições para a agregação nas dimensões/categorias, e conse- quentemente a estimação do indicador proposto (Índice de Sustentabilidade Hidroambiental para Bacias Hidrográficas - ISHBH).

De acordo com a proposta metodológica apresentada por Sepúlveda (2005), Waquil et. al. (2007) e Martins e Cândido (2008) quando as variáveis são escolhidas deve-se definir o tipo de relação que cada uma delas tem com o entorno geral. Segundo os autores, para cada variável, é necessário identificar se ela mede uma situação em que, ao aumentar seu valor, favorece ou desfavorece o processo de desenvolvimento, no caso do estudo de sustentabilidade hidroambiental. Assim, existe uma relação positiva se um aumento no valor da variável resulta em melhoria do sistema; em contrapartida, a relação é negativa se um aumento no valor da variável resulta em piora do sistema. A operacionalização é feita da seguinte forma (Quadro 1):

Quadro 1: Relação positiva e negativa para análise das variáveis

\begin{tabular}{|c|c|}
\hline $\begin{array}{c}\text { Relação Positiva: } \\
\mathrm{I}=\frac{\mathrm{x}-\mathrm{m}}{\mathrm{M}-\mathrm{m}}\end{array}$ & $\begin{array}{c}\text { Relação Negativa: } \\
\mathrm{I}=\frac{\mathrm{M}-\mathrm{x}}{\mathrm{M}-\mathrm{m}}\end{array}$ \\
\hline $\begin{array}{r}\text { Onde: } \mathrm{I}=\text { índice calculado referente a cada variável, para cada território analisado; } \mathrm{x}=\text { valor observado de cada variável em cada } \\
\text { território analisado; } \mathrm{m}=\text { valor mínimo considerado; } \mathrm{M}=\text { valor máximo considerado. }\end{array}$ \\
\hline
\end{tabular}

A definição do sinal, indicando se a relação é positiva ou negativa, foi feita a partir da intuição dos autores da pesquisa. Para tanto, foi realizado o cálculo do índice para cada indicador, e consequentemente realizado a agregação desses índices por dimensão através da média aritmética. Para a avaliação da sustentabilidade da sub-bacia foi utilizada a escala proposta por Guimarães, Turetta e Coutinho (2010), que varia de 0,00 a 1,00. Ela está dividida em cinco setores de 20 pontos cada com a seguinte classificação (Quadro 2):

Quadro 2: Classificação e representação dos índices em níveis de sustentabilidade hidroambiental

\begin{tabular}{|c|c|}
\hline Índice $\mathbf{( 0 , 0 0 - 1 , 0 0})$ & Desempenho \\
\hline $0,00-0,20$ & Ruim / insustentável \\
\hline $0,21-0,40$ & Pobre / potencialmente insustentável \\
\hline $0,41-0,60$ & Médio / intermediário \\
\hline $0,61-0,80$ & Bom / potencialmente sustentável \\
\hline $0,81-1,00$ & Muito bom / sustentável \\
\hline
\end{tabular}

Fonte: Adaptado para o estudo conforme Guimarães, Turetta e Coutinho (2010). 
Proposta e validação de indicadores hidroambientais para bacias hidrográficas: estudo de caso na sub-bacia do alto curso do Rio Paraíba, PB. José Ribamar Marques de Carvalho, Wilson Fadlo Curi, Enyedja Kerlly Martins de Araújo Carvalho, Rosires Catão Curi

A sub-bacia utilizada no estudo faz parte da bacia hidrográfica do Rio Paraíba (constituída por quatro sub-bacias, conforme destaque na figura 1). Para esse estudo foi selecionada a Sub-bacia do Alto Curso do Rio Paraíba, PB. Nesta sub-bacia estão loca- lizados 17 municípios: Amparo, Barra de São Miguel, Boqueirão, Cabaceiras, Camalaú, Congo, Coxixola, Monteiro, Ouro Velho, Prata, São Domingos do Cariri, São João do Cariri, São João do Tigre, São Sebastião do Umbuzeiro, Serra Branca, Sumé e Zabelê.

Figura 1: Localização da área de estudo - Região do Alto Curso do Rio Paraíba

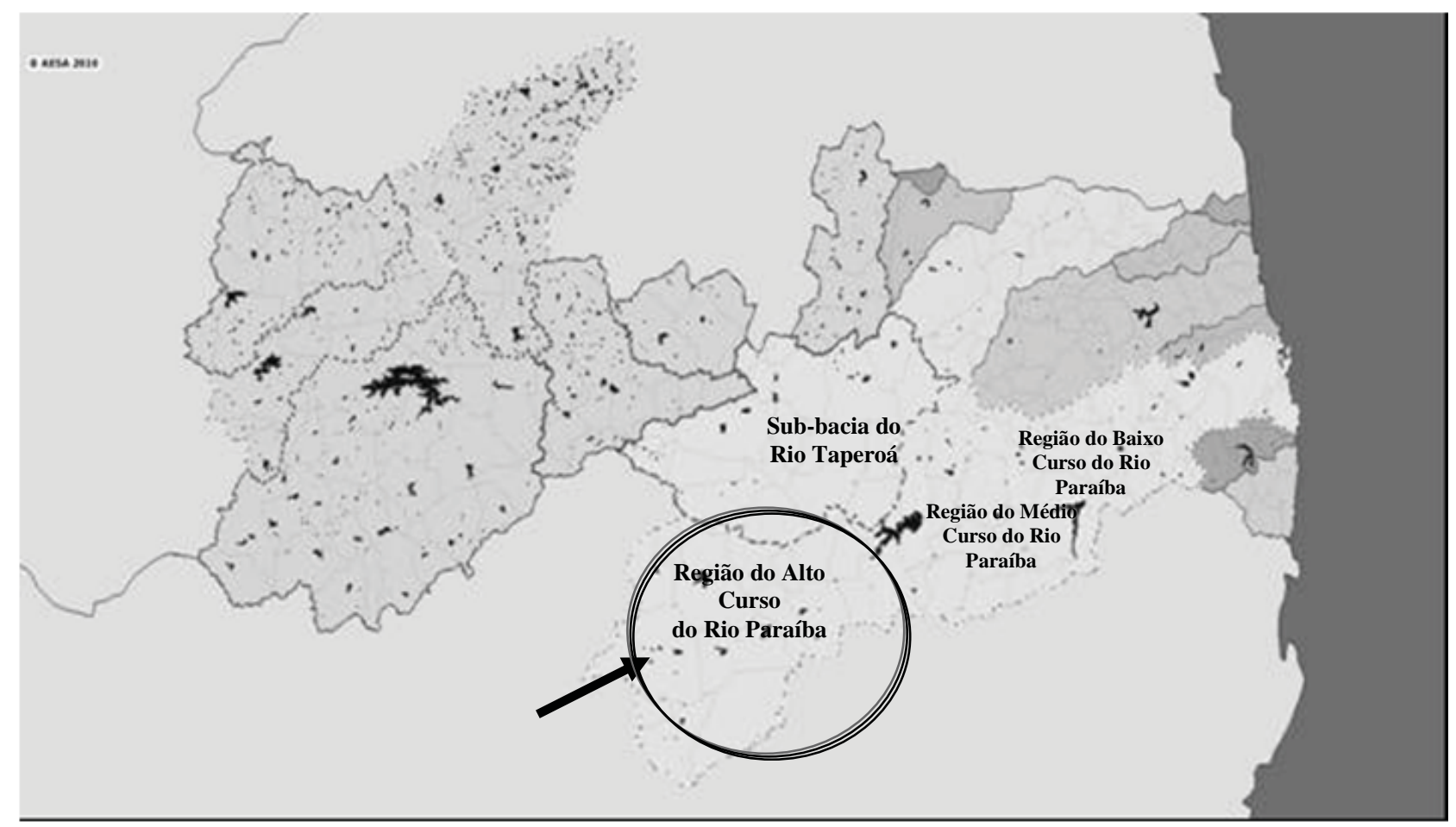

Fonte: AESA, 2011.

As categorias/dimensões foram distribuídas em 51 indicadores conforme descrição a seguir (Quadro 3): 
Proposta e validação de indicadores hidroambientais para bacias hidrográficas: estudo de caso na sub-bacia do alto curso do Rio Paraíba, PB. José Ribamar Marques de Carvalho, Wilson Fadlo Curi, Enyedja Kerlly Martins de Araújo Carvalho, Rosires Catão Curi

Quadro 3: Indicadores hidroambientais utilizados no estudo

\begin{tabular}{|c|c|c|c|}
\hline $\begin{array}{l}\text { CATEGORIA/ } \\
\text { DIMENSÃO }\end{array}$ & INDICADOR & FONTE & $\begin{array}{l}\text { TIPO DE RE- } \\
\text { LAÇÃO DO } \\
\text { INDICADOR }\end{array}$ \\
\hline \multirow{9}{*}{$\begin{array}{l}\text { DESEMPENHO DO } \\
\text { SISTEMA } \\
\text { QUANTO AO DE- } \\
\text { SENVOLVIMENTO } \\
\text { HUMANO }\end{array}$} & Taxa de Alfabetização & IDEME-PB (2008) & Positiva \\
\hline & Taxa de Mortalidade Infantil & IDEME-PB (2008) & Negativa \\
\hline & IDH-M (Índice de Desenvolvimento Humano Municipal) & IDEME-PB (2008) & Positiva \\
\hline & Expectativa de Vida ao Nascer & IDEME-PB (2008) & Positiva \\
\hline & Quantidade de estabelecimentos de saúde & IBGE (2009) & Positiva \\
\hline & Taxa de hospitalização por desidratação em menores de 5 anos & SIAB-DATASUS (2009) & Negativa \\
\hline & $\begin{array}{c}\text { Taxa de mortalidade infantil por diarreia } \\
\text { (por } 1.000 \text { nascidos vivos) }\end{array}$ & SIAB-DATASUS (2009) & Negativa \\
\hline & População coberta pelo Programa de Saúde da Família (PSF) & SIAB-DATASUS (2009) & Positiva \\
\hline & Relação entre população urbana e rural & IBGE $(2010)$ & Positiva \\
\hline \multirow{6}{*}{$\begin{array}{l}\text { DESEMPENHO } \\
\text { DO SISTEMA } \\
\text { QUANTOAOS } \\
\text { INDICADORES } \\
\text { ECONÔMICOS }\end{array}$} & PIB per capita em reais & IBGE (2008) & Positiva \\
\hline & Receitas de impostos e transferências de recursos & DATASUS (2009) & Positiva \\
\hline & Despesa total com saúde & SIOPS-DATASUS (2010) & Positiva \\
\hline & Despesa total com saúde por habitante & SIOPS-DATASUS (2010) & Positiva \\
\hline & Transferência SUS por habitante & SIOPS-DATASUS (2010) & Positiva \\
\hline & Tarifa Média de Água $\left(\mathrm{R} \$ / \mathrm{m}^{3}\right)$ & SNIS (2008) & Negativa \\
\hline \multirow{9}{*}{$\begin{array}{c}\text { DESEMPENHO } \\
\text { DO SISTEMA } \\
\text { QUANTO AO } \\
\text { ABASTECIMENTO } \\
\text { HUMANO }\end{array}$} & População total & IBGE (2010) & Positiva \\
\hline & Densidade demográfica & IBGE (2010) & Positiva \\
\hline & Precipitação média anual & AESA (2010) & Positiva \\
\hline & Consumo médio per capita (1/hab/dia) & SINS (2008) & Negativa \\
\hline & Índice de atendimento urbano de água (\%) & SINS (2008) & Positiva \\
\hline & Índice de perdas na distribuição (\%) & SINS (2008) & Negativa \\
\hline & $\%$ abastecimento por rede geral & DATASUS, IBGE (2002) & Positiva \\
\hline & $\%$ de abastecimento por poço nascente & DATASUS, IBGE (2002) & Negativa \\
\hline & $\%$ Outra forma de abastecimento de água & DATASUS, IBGE (2002) & Negativa \\
\hline \multirow{11}{*}{$\begin{array}{c}\text { DESEMPENHO } \\
\text { DO SISTEMA } \\
\text { QUANTO ĀS } \\
\text { PRESSÕES DA } \\
\text { IRRIGAÇÃO, } \\
\text { PECUÁRIA, } \\
\text { PBSTECIMENTO } \\
\text { RUURAL, } \\
\text { RURAL } \\
\text { AQUICULTURA E } \\
\text { LAZER }\end{array}$} & Outorga p/irrigação $\left(\mathrm{m}^{3} / \mathrm{h}\right)$ & AESA (2010) & Negativa \\
\hline & Outorga p/abast.rural $\left(\mathrm{m}^{3} / \mathrm{h}\right)$ & AESA (2010) & Negativa \\
\hline & Outorga $\mathrm{p} /$ abast. urbano $\left(\mathrm{m}^{3} / \mathrm{h}\right)$ & AESA (2010) & Negativa \\
\hline & Outro tipo de outorga $\left(\mathrm{m}^{3} / \mathrm{h}\right)$ & AESA (2010) & Negativa \\
\hline & Número de bovinos & IBGE (2009) & Negativa \\
\hline & Número de equinos & IBGE (2009) & Negativa \\
\hline & Número de assininos & IBGE (2009) & Negativa \\
\hline & Números de muares & IBGE (2009) & Negativa \\
\hline & Número de suínos & IBGE (2009) & Negativa \\
\hline & Número de caprinos & IBGE (2009) & Negativa \\
\hline & Número de ovinos & IBGE $(2009)$ & Negativa \\
\hline \multirow{6}{*}{$\begin{array}{c}\text { DESEMPENHO } \\
\text { DO SISTEMA } \\
\text { QUANTOA } \\
\text { COLETA DE } \\
\text { ESGOTOS }\end{array}$} & $\%$ rede sanitária via esgoto & DATASUS, IBGE (2002) & Positiva \\
\hline & $\%$ rede sanitária via fossa séptica & DATASUS, IBGE (2002) & Negativa \\
\hline & $\%$ rede sanitária via fossa rudimentar & DATASUS, IBGE (2002) & Negativa \\
\hline & $\%$ rede sanitária via vala & DATASUS, IBGE (2002) & Negativa \\
\hline & $\%$ rede sanitária via rio ou lago & DATASUS, IBGE (2002) & Negativa \\
\hline & \% que não dispõe de instalação sanitária & DATASUS, IBGE (2002) & Negativa \\
\hline \multirow{4}{*}{$\begin{array}{c}\text { DESEMPENHO DO } \\
\text { SISTEMA QUANTO } \\
\text { AO ATENDIMENTO } \\
\text { DE COLETA DE } \\
\text { LIXO } \\
\end{array}$} & $\%$ de lixo coletado & DATASUS, IBGE (2002) & Positiva \\
\hline & \% lixo queimado & DATASUS, IBGE (2002) & Negativa \\
\hline & $\%$ lixo enterrado & DATASUS, IBGE (2002) & Negativa \\
\hline & \% lixo jogado & DATASUS, IBGE (2002) & Negativa \\
\hline \multirow{6}{*}{$\begin{array}{l}\text { DESEMPENHO } \\
\text { DO SISTEMA } \\
\text { QUANTO AO } \\
\text { ESTADO } \\
\text { QUALITATIVO } \\
\text { DA ÁGUA }\end{array}$} & Incidência das análises de cloro residual fora do padrão (\%) & SNIS (2008) & Negativa \\
\hline & Incidência das análises de turbidez fora do padrão (\%) & SNIS (2008) & Negativa \\
\hline & $\begin{array}{c}\text { Índice de conformidade da quantidade de amostras - } \\
\text { cloro residual (\%) }\end{array}$ & SNIS (2008) & Negativa \\
\hline & $\begin{array}{c}\text { Indice de conformidade da quantidade de amostras - } \\
\text { turbidez }(\%)\end{array}$ & SNIS (2008) & Negativa \\
\hline & Incidência das análises de coliformes totais fora do padrão (\%) & SNIS (2008) & Negativa \\
\hline & $\begin{array}{c}\text { Índice de conformidade da quantidade de amostras - } \\
\text { coliformes totais }(\%)\end{array}$ & SNIS (2008) & Negativa \\
\hline
\end{tabular}

Fonte: Elaboração própria, 2011. 
Proposta e validação de indicadores hidroambientais para bacias hidrográficas: estudo de caso na sub-bacia do alto curso do Rio Paraíba, PB. José Ribamar Marques de Carvalho, Wilson Fadlo Curi, Enyedja Kerlly Martins de Araújo Carvalho, Rosires Catão Curi

\section{RESULTADOS E DISCUSSÃO}

A seguir estão evidenciados os resultados relacionados à caracterização dos indicadores de cada uma das dimensões analisadas e consideradas no estudo. A análise foi feita de forma comparativa entre os dezessete municípios analisados e que fazem parte da sub-bacia
Alto Curso do Rio Paraíba (Bacia do Rio Paraíba).

Após a ponderação dos indicadores relacionados ao desempenho do sistema quanto ao desenvolvimento humano, apresentados no Quadro 3, de cada município e após as análises positivas e negativas de cada um deles foi possível chegar aos resultados que estão evidenciados na Tabela 1.

Tabela 1: Desempenho do Sistema quanto ao Desenvolvimento Humano

\begin{tabular}{ccc}
\hline Municípios & Média & Desempenho \\
\hline Amparo & $\mathbf{0 , 4 6}$ & Intermediário \\
Barra de São Miguel & $\mathbf{0 , 4 3}$ & Intermediário \\
Boqueirão & $\mathbf{0 , 6 3}$ & Potencialmente sustentável \\
Cabaceiras & $\mathbf{0 , 6 2}$ & Potencialmente sustentável \\
Camalaú & $\mathbf{0 , 4 1}$ & Intermediário \\
Congo & $\mathbf{0 , 5 4}$ & Intermediário \\
Coxixola & $\mathbf{0 , 3 5}$ & Potencialmente insustentável \\
Monteiro & $\mathbf{0 , 4 9}$ & Intermediário \\
Ouro Velho & $\mathbf{0 , 5 0}$ & Intermediário \\
Prata & 0,41 & Intermediário \\
São Domingos do Cariri & $\mathbf{0 , 6 4}$ & Potencialmente sustentável \\
São João do Cariri & $\mathbf{0 , 5 7}$ & Intermediário \\
São João do Tigre & $\mathbf{0 , 2 3}$ & Potencialmente insustentável \\
São Sebastião do Umbuzeiro & $\mathbf{0 , 3 8}$ & Potencialmente insustentável \\
Serra Branca & $\mathbf{0 , 6 3}$ & Potencialmente sustentável \\
Sumé & $\mathbf{0 , 6 8}$ & Potencialmente sustentável \\
Zabelê & $\mathbf{0 , 5 4}$ & Intermediário \\
\hline Média Geral & 0,50 & Intermediário \\
\hline
\end{tabular}

Fonte: Dados da pesquisa, 2011.

Observa-se que dos dezessete municípios estudados, cinco cidades apresentaram uma situação potencialmente sustentável: Boqueirão $(0,63)$, Cabaceiras $(0,62)$, São Domingos do Cariri $(0,64)$, Serra Branca $(0,63)$ e Sumé com o melhor desempenho $(0,68)$. Outros nove municípios apresentaram desempenho intermediário: Amparo $(0,46)$, Barra de São Miguel $(0,43)$, Camalaú $(0,41)$, Congo $(0,54)$, Monteiro $(0,49)$, Ouro Velho $(0,50)$, Prata $(0,41)$, São João do Cariri $(0,57)$ e Zabelê $(0,54)$. Três municípios apresentaram situação potencialmente insustentável: Coxixola $(0,35)$, São João do Rio Tigre $(0,23)$ com a pior situação e São Sebastião do Umbuzeiro $(0,38)$. Verifica-se que dentre os 17 municípios estudados, nenhum deles apresentou situação insustentável.

A média geral dos municípios localizados na sub-bacia é aceitável $(0,53)$ na ótica do desenvolvimento humano, todavia, fica evidente a necessidade de melhorar essa situação em busca da situação ideal de modo que possa contribuir satisfatoriamente para esse contexto geográfico.

Prosseguindo na análise, passa-se ao desempenho do sistema quanto aos indicadores econômicos, segundo os parâmetros adotados, apenas o município de Monteiro apresentou a situação potencialmente sustentável $(0,64)$, se comparado com os demais municípios, seguido de Sumé $(0,44)$ com situação intermediária. A grande maioria dos municípios 
Proposta e validação de indicadores hidroambientais para bacias hidrográficas: estudo de caso na sub-bacia do alto curso do Rio Paraíba, PB. José Ribamar Marques de Carvalho, Wilson Fadlo Curi, Enyedja Kerlly Martins de Araújo Carvalho, Rosires Catão Curi

apresentou situação insustentável ou potencialmente insustentável (70,59\%). Talvez uma das causas desse rendimento crítico esteja relacionada à grande dependência financeira que esses municípios detêm em relação a transferências intergovernamentais, ou seja, os entraves financeiros são sanados apenas com os recursos oriundos do governo federal, o que demonstra que os anseios da sociedade podem não ser atingidos com os atuais patamares de recursos. Ainda não existe nessas comunidades uma política voltada para a geração de renda a partir das potencialidades locais, denotando um falta de política sustentável em relação a essa categoria. Por conta desse cenário a média geral da categoria dos municípios da sub-bacia apresentou um nível potencialmente insustentável o que certamente influencia a adoção de práticas e iniciativas relacionadas aos recursos hídricos (Tabela 2).

Tabela 2: Desempenho do Sistema quanto aos Indicadores Econômicos

\begin{tabular}{ccc}
\hline Municípios & Média & Desempenho \\
\hline Amparo & $\mathbf{0 , 3 6}$ & Potencialmente insustentável \\
Barra de São Miguel & $\mathbf{0 , 0 8}$ & Insustentável \\
Boqueirão & $\mathbf{0 , 4 3}$ & Intermediário \\
Cabaceiras & $\mathbf{0 , 1 2}$ & Insustentável \\
Camalaú & $\mathbf{0 , 1 9}$ & Insustentável \\
Congo & $\mathbf{0 , 2 7}$ & Intermediário \\
Coxixola & $\mathbf{0 , 3 0}$ & Intermediário \\
Monteiro & $\mathbf{0 , 6 4}$ & Potencialmente sustentável \\
Ouro Velho & $\mathbf{0 , 2 1}$ & Potencialmente insustentável \\
Prata & $\mathbf{0 , 3 5}$ & Potencialmente insustentável \\
São Domingos do Cariri & $\mathbf{0 , 2 2}$ & Potencialmente insustentável \\
São João do Cariri & $\mathbf{0 , 1 5}$ & Insustentável \\
São João do Tigre & $\mathbf{0 , 0 6}$ & Insustentável \\
São Sebastião do Umbuzeiro & $\mathbf{0 , 1 4}$ & Insustentável \\
Serra Branca & $\mathbf{0 , 3 4}$ & Potencialmente insustentável \\
Sumé & $\mathbf{0 , 4 4}$ & Intermediário \\
Zabelê & $\mathbf{0 , 2 6}$ & Potencialmente insustentável \\
\hline Média & 0,27 & Potencialmente insustentável \\
\hline
\end{tabular}

Fonte: Dados da pesquisa, 2011.

Em relação à categoria abastecimento humano (Tabela 3), o indicador foi constituído levando-se em consideração os parâmetros mostrados no Quadro 1. Percebe-se, conforme a tabela 3, que o desempenho dos indicadores dessa categoria se apresentaram da seguinte forma: $17,65 \%$ tiveram uma avaliação potencialmente sustentável (Boqueirão - 0,71, Monteiro e Ouro Velho - 0,73 respectivamente); 23,53\% tiveram uma avaliação potencialmente insustentável (Amparo - 0,25, Barra de São Miguel - 0,31, São Sebastião do
Umbuzeiro 0,38 e Zabelê 0,34); 58,82\% tiveram uma avaliação intermediária (Cabaceiras - 0,43, Camalaú0,47, Congo - 0,52, Coxixola - 0,49, Prata $-0,57$, São Domingos do Cariri 0,47, São João do Cariri - 0, 47, Serra Branca - 0,46 e Sumé - 0,57); e 5,88\% obteve uma avaliação insustentável (São João do Rio Tigre $0,23)$. No geral a média dessa categoria foi igual a 0,47 demonstrando um desempenho intermediário e que precisa de esforços na tentativa de melhorar esta performance. 
Proposta e validação de indicadores hidroambientais para bacias hidrográficas: estudo de caso na sub-bacia do alto curso do Rio Paraíba, PB. José Ribamar Marques de Carvalho, Wilson Fadlo Curi, Enyedja Kerlly Martins de Araújo Carvalho, Rosires Catão Curi

Tabela 3: Desempenho do Sistema quanto ao Abastecimento Humano

\begin{tabular}{ccc}
\hline Municípios & Média & Desempenho \\
\hline Amparo & $\mathbf{0 , 2 5}$ & Potencialmente insustentável \\
Barra de São Miguel & $\mathbf{0 , 3 1}$ & Potencialmente insustentável \\
Boqueirão & $\mathbf{0 , 7 1}$ & Potencialmente sustentável \\
Cabaceiras & $\mathbf{0 , 4 3}$ & Intermediário \\
Camalaú & $\mathbf{0 , 4 7}$ & Intermediário \\
Congo & $\mathbf{0 , 5 2}$ & Intermediário \\
Coxixola & $\mathbf{0 , 4 9}$ & Intermediário \\
Monteiro & $\mathbf{0 , 7 3}$ & Potencialmente sustentável \\
Ouro Velho & $\mathbf{0 , 7 3}$ & Potencialmente sustentável \\
Prata & $\mathbf{0 , 5 7}$ & Intermediário \\
São Domingos do Cariri & $\mathbf{0 , 4 7}$ & Intermediário \\
São João do Cariri & $\mathbf{0 , 4 7}$ & Intermediário \\
São João do Tigre & $\mathbf{0 , 2 0}$ & Insustentável \\
São Sebastião do Umbuzeiro & $\mathbf{0 , 3 8}$ & Potencialmente insustentável \\
Serra Branca & $\mathbf{0 , 4 6}$ & Intermediário \\
Sumé & $\mathbf{0 , 5 7}$ & Intermediário \\
Zabelê & $\mathbf{0 , 3 4}$ & Potencialmente insustentável \\
\hline Média & $\mathbf{0 , 4 7}$ & Intermediário \\
\hline
\end{tabular}

Fonte: Dados da pesquisa, 2011.

O índice de sustentabilidade hidroambiental desta categoria (conforme os parâmetros definidos no Quadro 1) foi de 0,76, onde mostra uma situação potencialmente sustentável em 35,29\% dos municípios, $52,94 \%$ em situação sustentável e 11,76\% em situação intermediária e insustentável (Sumé e Monteiro). Na Tabela 4 é possível visualizar o panorama dos indicadores, com os respectivos índices de sustentabilidade hidroambiental para essa categoria. Esse resultado advém do fato de que nessas localidades as atividades econômicas principais não estão relacionadas a estas pressões (pecuária, irrigação, abastecimento rural, aquicultura e lazer), o que minimiza os impactos sobre os recursos hídricos. Observe que a situação desses municípios (Sumé e Monteiro) se configura como uma situação de alerta posto que existe uma forte pressão exercida fruto das outorgas concedidas e da concentração de atividades relacionadas a pecuária.

No que se refere à categoria relacionada ao desempenho do sistema de quanto à coleta de esgotos (Tabela 5) verifica-se que a média geral deste indicador apresentou resultado potencialmente sustentável, uma vez que seu índice de sustentabilidade hidroambiental foi de 0,65 . Cerca de 70,59\% dos municípios apresentaram situação dentro dos parâmetros aceitáveis (potencialmente sustentável e sustentável). Destaque ainda que $29,41 \%$ dos municípios apresentou uma performance intermediária (indicadores entre 0,41 e 0,60 ), mostrando que muito ainda precisa ser feito para melhorar essa dimensão.

Como essas variáveis são tidas como elementos que exercem forte pressão hídrica e apresentam números abaixo dos ideais, esses indicadores demonstram a necessidade de definição de políticas dos gestores públicos dos municípios analisados, mesmo em um cenário aceitável, em busca de manter ou melhorar tais resultados, sendo necessário maior investimento nesse tipo de infraestrutura. 
Proposta e validação de indicadores hidroambientais para bacias hidrográficas: estudo de caso na sub-bacia do alto curso do Rio Paraíba, PB. José Ribamar Marques de Carvalho, Wilson Fadlo Curi, Enyedja Kerlly Martins de Araújo Carvalho, Rosires Catão Curi

Tabela 4: Desempenho do sistema quanto às pressões da irrig. pec, abastecimento rural, aquicultura e lazer

\begin{tabular}{ccc}
\hline Municípios & Média & Desempenho \\
\hline Amparo & $\mathbf{0 , 9 1}$ & Sustentável \\
Barra de São Miguel & $\mathbf{0 , 8 1}$ & Sustentável \\
Boqueirão & $\mathbf{0 , 7 3}$ & Potencialmente sustentável \\
Cabaceiras & $\mathbf{0 , 7 3}$ & Potencialmente sustentável \\
Camalaú & $\mathbf{0 , 6 6}$ & Potencialmente sustentável \\
Congo & $\mathbf{0 , 7 3}$ & Potencialmente sustentável \\
Coxixola & $\mathbf{0 , 9 2}$ & Sustentável \\
Monteiro & $\mathbf{0 , 3 5}$ & Potencialmente insustentável \\
Ouro Velho & $\mathbf{0 , 9 5}$ & Sustentável \\
Prata & $\mathbf{0 , 8 8}$ & Sustentável \\
São Domingos do Cariri & $\mathbf{0 , 8 1}$ & Sustentável \\
São João do Cariri & $\mathbf{0 , 8 1}$ & Sustentável \\
São João do Tigre & 0,73 & Potencialmente sustentável \\
São Sebastião do Umbuzeiro & $\mathbf{0 , 8 2}$ & Sustentável \\
Serra Branca & $\mathbf{0 , 6 5}$ & Potencialmente sustentável \\
Sumé & $\mathbf{0 , 5 8}$ & Intermediário \\
Zabelê & $\mathbf{0 , 9 1}$ & Sustentável \\
\hline Média & $\mathbf{0 , 7 6}$ & Potencialmente sustentável \\
\hline
\end{tabular}

Fonte: Dados da pesquisa, 2011.

Tabela 5: Desempenho do sistema quanto à coleta de esgotos

\begin{tabular}{ccc}
\hline Municípios & Média & Desempenho \\
\hline Amparo & $\mathbf{0 , 5 9}$ & Intermediário \\
Barra de São Miguel & $\mathbf{0 , 5 4}$ & Intermediário \\
Boqueirão & $\mathbf{0 , 8 4}$ & Sustentável \\
Cabaceiras & $\mathbf{0 , 8 1}$ & Sustentável \\
Camalaú & $\mathbf{0 , 6 1}$ & Potencialmente sustentável \\
Congo & $\mathbf{0 , 7 0}$ & Potencialmente sustentável \\
Coxixola & $\mathbf{0 , 7 8}$ & Potencialmente sustentável \\
Monteiro & $\mathbf{0 , 8 6}$ & Sustentável \\
Ouro Velho & $\mathbf{0 , 6 1}$ & Potencialmente sustentável \\
Prata & $\mathbf{0 , 6 2}$ & Potencialmente sustentável \\
São Domingos do Cariri & $\mathbf{0 , 5 2}$ & Intermediário \\
São João do Cariri & $\mathbf{0 , 5 1}$ & Intermediário \\
São João do Tigre & $\mathbf{0 , 6 5}$ & Potencialmente sustentável \\
São Sebastião do Umbuzeiro & $\mathbf{0 , 5 6}$ & Intermediário \\
Serra Branca & $\mathbf{0 , 6 4}$ & Potencialmente sustentável \\
Sumé & $\mathbf{0 , 6 6}$ & Potencialmente sustentável \\
Zabelê & $\mathbf{0 , 7 6}$ & Potencialmente sustentável \\
\hline Média & $\mathbf{0 , 6 5}$ & Potencialmente sustentável \\
\hline
\end{tabular}

Fonte: Dados da pesquisa, 2011. 
Proposta e validação de indicadores hidroambientais para bacias hidrográficas: estudo de caso na sub-bacia do alto curso do Rio Paraíba, PB. José Ribamar Marques de Carvalho, Wilson Fadlo Curi, Enyedja Kerlly Martins de Araújo Carvalho, Rosires Catão Curi

Em relação à categoria desempenho do sistema quanto ao atendimento de coleta de lixo (Tabela 6) contatou-se a seguinte desempenho para seus indicadores: 29,41\% dos municípios apresentaram uma potencialmente insustentável, 35,29\% tiveram uma avaliação intermediária. Os municípios com melhores performance foram: São Sebastião do Umbuzeiro $(0,89)$ e Sumé $(0,87)$. Notadamente, os piores desempenhos foram obtidos pelos seguintes municípios: Amparo (0,28), São Domingos do Cariri $(0,26)$, São João do Tigre $(0,31)$, Cabaceiras $(0,36)$ e Barra de São Miguel $(0,36)$.

Tabela 6: Desempenho do sistema quanto ao atendimento de coleta de lixo

\begin{tabular}{ccc}
\hline Municípios & Média & Desempenho \\
\hline Amparo & $\mathbf{0 , 2 8}$ & Potencialmente insustentável \\
Barra de São Miguel & $\mathbf{0 , 3 6}$ & Potencialmente insustentável \\
Boqueirão & $\mathbf{0 , 7 7}$ & Potencialmente sustentável \\
Cabaceiras & $\mathbf{0 , 3 6}$ & Potencialmente insustentável \\
Camalaú & $\mathbf{0 , 5 9}$ & Intermediário \\
Congo & $\mathbf{0 , 4 1}$ & Intermediário \\
Coxixola & $\mathbf{0 , 4 7}$ & Intermediário \\
Monteiro & $\mathbf{0 , 7 3}$ & Potencialmente sustentável \\
Ouro Velho & $\mathbf{0 , 7 8}$ & Potencialmente sustentável \\
Prata & $\mathbf{0 , 5 8}$ & Intermediário \\
São Domingos do Cariri & $\mathbf{0 , 2 6}$ & Potencialmente insustentável \\
São João do Cariri & $\mathbf{0 , 4 3}$ & Intermediário \\
São João do Tigre & $\mathbf{0 , 3 1}$ & Potencialmente insustentável \\
São Sebastião do Umbuzeiro & $\mathbf{0 , 8 9}$ & Sustentável \\
Serra Branca & $\mathbf{0 , 5 9}$ & Intermediário \\
Sumé & $\mathbf{0 , 8 7}$ & Sustentável \\
Zabelê & $\mathbf{0 , 7 4}$ & Potencialmente sustentável \\
\hline Média & $\mathbf{0 , 5 5}$ & Intermediário \\
\hline
\end{tabular}

Fonte: Dados da pesquisa, 2011.

O saldo desta categoria não foi muito satisfatório, uma vez que o seu índice de sustentabilidade foi de 0,55 , um nível considerado em estado intermediário. Tal resultado demonstra que os municípios localizados nesta sub-bacia hidrográfica necessitam rever algumas alternativas para melhoria em termos de coleta de lixo, posto que essa dimensão exerce forte pressão sobre os recursos hídricos.

No que se refere ao desempenho do sistema quanto ao estado qualitativo da água (Tabela 7), foi possível observar o município que apresentar o pior desempenhos (Cabaceiras - 0,34). Os municípios de Coxixola $(0,81)$, Monteiro e Ouro Velho $(0,86)$ e Serra Branca $(0,95)$ apresentaram situação sustentável. Percebeu-se ainda que $47,06 \%$ das cidades estudadas podem ser classificados como potencialmente sustentáveis. No geral, os municípios apresentaram níveis intermediários, visto que, a maioria obteve média geral igual a 0,74 , o que denota níveis razoáveis em relação à qualidade da água disponibilizada pela rede geral da concessionária estadual (Companhia de Água e Esgotos da Paraíba - CAGEPA - PB).

No intuito de sintetizar os resultados obtidos com a análise, encontram-se explicitadas no Gráfico 1, o desempenho da sub-bacia por categoria/dimensão e os respectivos índices de sustentabilidade hidroambiental. Quatro categorias apresentaram situação mais desfavorável, quando analisados sob a perspectiva da sustentabilidade hidroambiental: econômica $(0,27)$, abastecimento humano $(0,47)$, categoria desenvolvi- 
Proposta e validação de indicadores hidroambientais para bacias hidrográficas: estudo de caso na sub-bacia do alto curso do Rio Paraíba, PB. José Ribamar Marques de Carvalho, Wilson Fadlo Curi, Enyedja Kerlly Martins de Araújo Carvalho, Rosires Catão Curi

mento humano $(0,50)$ e coleta de lixo $(0,55)$, sinalizando as dimensões que precisam ser melhoradas de modo que não possa agravar ainda mais a situação hídrica da região estudada. De outro modo as categorias/dimen- sões que apresentaram melhores desempenhos foram: pressões sobre os recursos hídricos $(0,76)$, estado qualitativo da água $(0,74)$ e coleta de esgotos $(0,65)$.

Tabela 7: Desempenho do sistema quanto ao estado qualitativo da água

\begin{tabular}{ccc}
\hline Municípios & Média & Desempenho \\
\hline Amparo & Sem dados & Sem dados \\
Barra de São Miguel & $\mathbf{0 , 6 6}$ & Potencialmente sustentável \\
Boqueirão & $\mathbf{0 , 7 4}$ & Potencialmente sustentável \\
Cabaceiras & $\mathbf{0 , 3 4}$ & Potencialmente insustentável \\
Camalaú & $\mathbf{0 , 7 1}$ & Potencialmente sustentável \\
Congo & $\mathbf{0 , 7 7}$ & Potencialmente sustentável \\
Coxixola & $\mathbf{0 , 8 1}$ & Sustentável \\
Monteiro & $\mathbf{0 , 8 6}$ & Sustentável \\
Ouro Velho & $\mathbf{0 , 8 6}$ & Sustentável \\
Prata & $\mathbf{0 , 7 0}$ & Potencialmente sustentável \\
São Domingos do Cariri & $\mathbf{0 , 6 9}$ & Potencialmente sustentável \\
São João do Cariri & $\mathbf{0 , 7 6}$ & Potencialmente sustentável \\
São João do Tigre & Sem dados & Sem dados \\
São Sebastião do Umbuzeiro & Sem dados & Sem dados \\
Serra Branca & $\mathbf{0 , 9 5}$ & Sustentável \\
Sumé & $\mathbf{0 , 8 0}$ & Potencialmente sustentável \\
Zabelê & Sem dados & Sem dados \\
\hline Média & $\mathbf{0 , 7 4}$ & Potencialmente sustentável \\
\hline
\end{tabular}

Fonte: Dados da pesquisa, 2011.

Gráfico 1: Biograma geral das categorias/dimensões analisadas

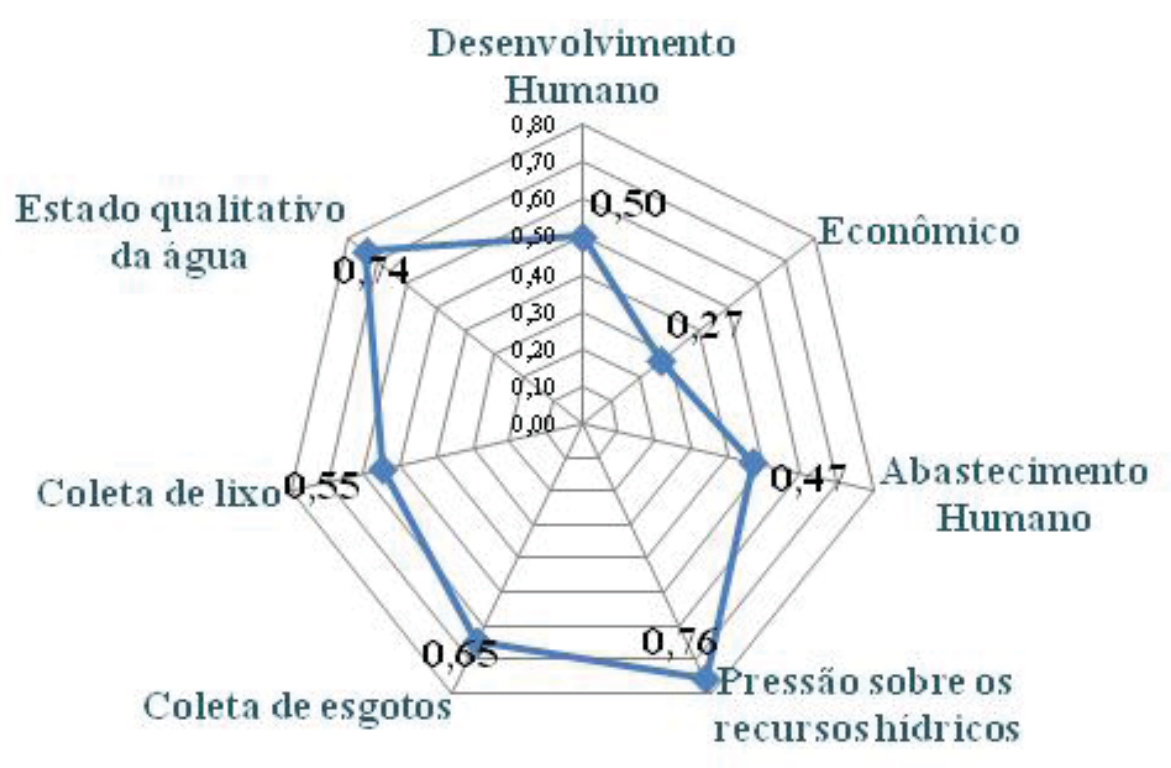

Fonte: Dados da pesquisa, 2011. 
Finalmente, a partir do biograma apresentado no Gráfico 2 é possível visualizar melhor os níveis de sustentabilidade hidroambiental dos municípios analisados. Os resultados obtidos apontam que os municípios com melhores performances hidroambiental foram: Boqueirão $(0,69)$, Monteiro $(0,67)$, Sumé e Ouro Velho $(0,66)$, Serra Branca $(0,61)$. Os municípios com piores desempenhos foram: Prata e Coxixola $(0,59)$, Congo $(0,56)$, São João do Cariri $(0,53)$, Camalaú e São Domingos do Cariri $(0,52)$, Zabelê $(0,51)$, Cabaceiras (0,49), Barra de São Miguel $(0,46)$, São Sebastião do Umbuzeiro $(0,45)$, Amparo $(0,41)$ e São João do Tigre $(0,31)$.

Gráfico 2: Biograma do ISHBH dos Municípios da Sub-bacia Hidrográfica

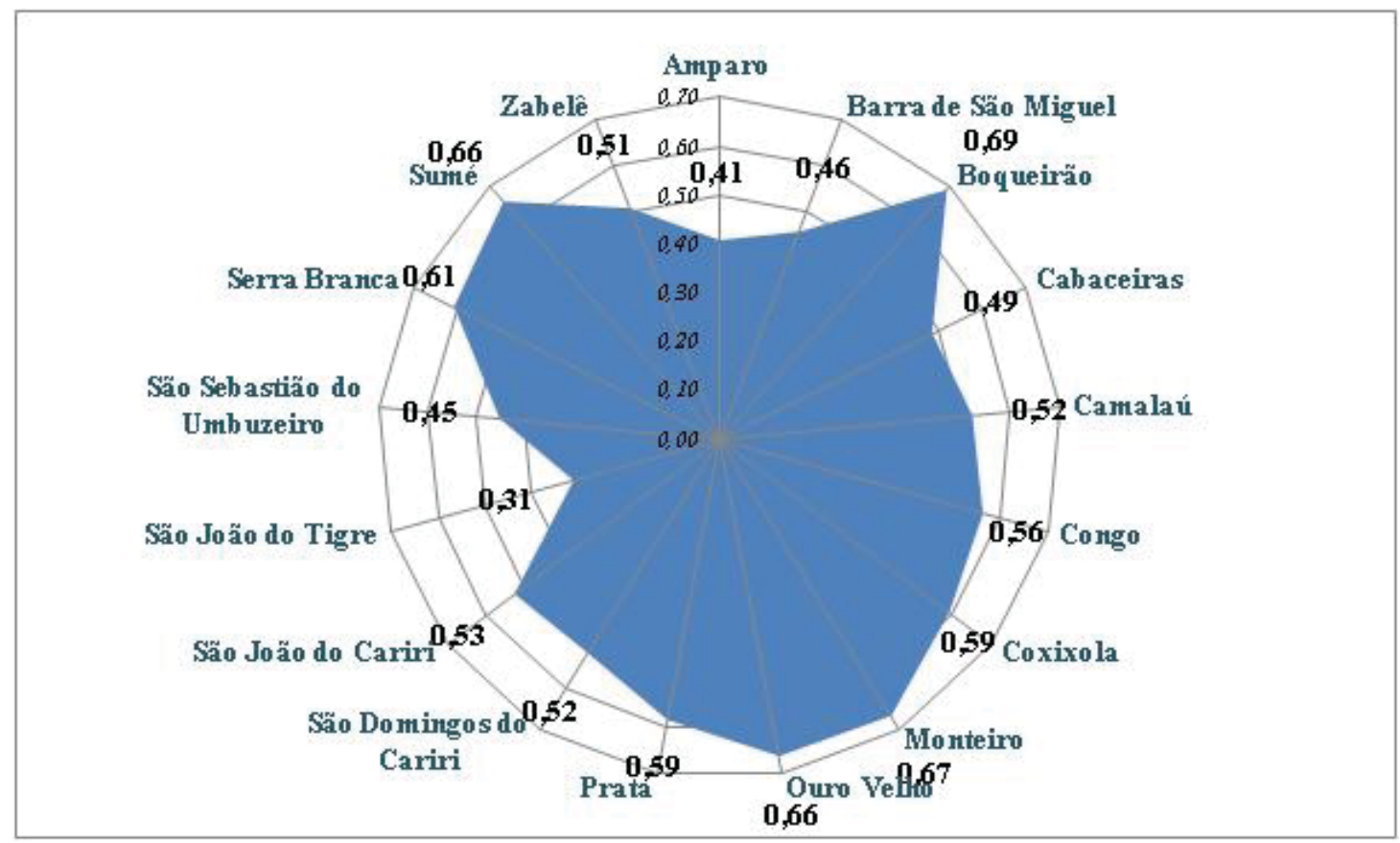

Fonte: Dados da pesquisa, 2011.

Esses resultados demonstram a situação de insustentabilidade hídrica que alguns municípios enfrentam, evidenciando a necessidade de definir programas de gestão hídrica capazes de reverter esse cenário de instabilidade, através de ações mais responsáveis por parte das entidades reguladoras da gestão de águas e demais entidades de classe no estado paraibano e em especial na sub-bacia estudada.

\section{CONSIDERAÇÕES FINAIS}

A realização deste estudo permitiu verificar o nível de sustentabilidade da sub-bacia hidrográfica do Alto Curso do Rio Paraíba, PB, evidenciando quais municípios se encontram em situação menos favorável (insustentável) em relação à sustentabilidade hídrica, segundo a escala adotada no estudo.

De maneira geral pode-se afirmar que os aspectos relacionados às dimensões/categorias econômicas, coleta de lixo e desenvolvimento humano são as que necessitam de mais ações com vistas a melhorias, uma vez que nelas residem entraves consideráveis relacionados à sustentabilidade hídrica da sub-bacia estudada.

Notadamente, faz-se necessário buscar alternativas que possam determinar o êxito desse cenário, sendo essencial a integração e a inclusão dos diferentes atores sociais no processo de forma que possa ser possível buscar alternativas capazes de reverter esse cenário.

Destaque-se também a necessidade de definir políticas públicas no sentido de estimular o crescimen- 
Proposta e validação de indicadores hidroambientais para bacias hidrográficas: estudo de caso na sub-bacia do alto curso do Rio Paraíba, PB. José Ribamar Marques de Carvalho, Wilson Fadlo Curi, Enyedja Kerlly Martins de Araújo Carvalho, Rosires Catão Curi

to da geração de alternativas sustentáveis de emprego e renda, dos níveis de educação ambiental, bem como em investimentos nas áreas diretamente relacionadas aos recursos hídricos, na tentativa de vislumbrar resultados mais satisfatórios.

Como sugestão para trabalhos futuros, sugere-se a ampliação do número de informações (dados) relativo aos parâmetros importantes para a análise do sistema, assim como o número de indicadores e a aplicação em outras sub-bacias ou bacias hidrográficas do estado Paraibano, bem como de outros estados de maneira que possa dispor de critérios mais amplos em relação à sustentabilidade hidroambiental.

Finalmente, pode-se inferir e reconhecer que os resultados referentes à aplicação dessa metodologia para construção de um índice de sustentabilidade hidroambiental para bacias hidrográficas possibilita obter informações relevantes acerca da realidade estudada, através da mensuração de indicadores que forneceram informações para a identificação das dimensões/categorias analisadas. Todavia, e considerando os argumentos destacados por Cândido (2010), qualquer tentativa de medir o desenvolvimento e a sustentabilidade tem sempre muitas limitações e serão sempre passíveis de discussões, uma vez que se trata de questões complexas e paradoxais.

As limitações do estudo giram em torno de aspectos relacionados à complexidade de cada um dos indicadores, variáveis e dimensões/categorias envolvidas; pela multiplicidade de possibilidades de inter-relações entre os indicadores considerados; pela falta de bancos de dados fidedignos e, ainda, por outras razões diversas que um ou outro indicador não foi utilizado. Além disso, existe a percepção diferenciada dos vários atores sociais e das institucionais envolvidas, que são reflexos de valores culturais, econômicos, políticos, institucionais a respeito de tais indicadores e sua relação em um processo de desenvolvimento sustentável.

\section{REFERÊNCIAS}

INSTITUTO DE DESENVOLVIMENTO MUNICIPALE ESTADUAL - IDEME/PB. Anuário Estatístico da Paraíba. João Pessoa: IDEME, 2008.
AGÊNCIA ESTADUAL DE ÁGUAS DA PARAÍBA-AESA. Disponível em: <www.aesa.pb.gov.br>. Acesso em: 29 fev. 2011.

CADERNO DE INFORMAÇÕES SOBRE SAÚDE DA PARAÍBA. Informações de Saúde - datasus. Disponível em: $<$ http://tabnet.datasus.gov.br/tabdata/ cadernos/pb.htm>. Acesso em: 10 mar. 2011.

CÂNDIDO, G. A. Anotações de aula: Disciplina Sistema de Indicadores de Sustentabilidade. 2010. Tese (Doutorado) - Programa de Pós-Graduação em Recursos Naturais - Universidade Federal de Campina Grande, Campina Grande 2010.

DANIEL, O. Definição de Indicadores de Sustentabilidade para Sistemas Agroflorestais. 2000. 123 p. Tese (Doutorado) - Programa de Pós-Graduação em Ciência Florestal - Universidade Federal de Viçosa, Viçosa, 2000.

BRASIL. Sistema Nacional de Informações sobre Saneamento (SNIS). Diagnóstico dos Serviços de Água e Esgotos. 2008. Disponível em: <http://www.snis.gov. br/PaginaCarrega.php?EWRErterterTERTer=85>. Acesso em: 15 mar. 2011.

KRONEMBERGER, D. M. P. et al. Desenvolvimento Sustentável no Brasil: Uma Análise a partir da Aplicação do Barômetro da Sustentabilidade. Revista Sociedade \& Natureza, Uberlândia, v. 20, n. 1, p. 25-50, jun. 2008.

GUIMARÃES, L. T. Proposta de um Sistema de Indicadores de Desenvolvimento Sustentável para Bacias Hidrográficas. 2008. 253 p. Tese (Doutorado) - Programa de Pós-graduação de Engenharia da Universidade Federal do Rio de Janeiro, Rio de Janeiro, 2008.

GUIMARÃES, L. T.; TURETTA, A. P. D.; COUTINHO, H. L. C. Uma proposta para avaliar a sustentabilidade da expansão do cultivo da cana-de-açúcar no Estado do Mato Grosso do Sul. Revista Sociedade \& Natureza, Uberlândia, v. 22, n. 2, p. 313-327, ago. 2010. 
Proposta e validação de indicadores hidroambientais para bacias hidrográficas: estudo de caso na sub-bacia do alto curso do Rio Paraíba, PB. José Ribamar Marques de Carvalho, Wilson Fadlo Curi, Enyedja Kerlly Martins de Araújo Carvalho, Rosires Catão Curi

INSTITUTO BRASILEIRO DE GEOGRAFIA E ESTATÍSTICA. Disponível em:<www.ibge.gov.br>. Acesso em: 29 fev. 2011

LIRA, W. S. Sistema de Gestão do Conhecimento para Indicadores de Sustentabilidade - SIGECIS: Proposta de uma metodologia. Campina Grande PB. 2008. 178 p. Tese (Doutorado) - Programa de Pós-Graduação em Recursos Naturais, Universidade Federal de Campina Grande. Campina Grande, 2008.

MAGALHÃES JÚNIOR, A. P. Indicadores Ambientais e Recursos Hídricos: Realidade e Perspectivas para o Brasil a partir da Experiência Francesa. 2. ed. Bertrand Brasil, 2010, 636 p.

MARANHÃO, N. Sistema de Indicadores para Planejamento e Gestão dos Recursos Hídricos de Bacias Hidrográficas. Tese (Doutorado) - Programa de Pós-Graduação de Engenharia Civil da Universidade Federal do Rio de Janeiro, 2007, p.422.

MARTINS, M. F.; CÂNDIDO, G. A. Índice de Desenvolvimento Sustentável para Municípios (IDSM): metodologia para análise e cálculo do IDSM e classificação dos níveis de sustentabilidade - uma aplicação no Estado da Paraíba. João Pessoa: Sebrae, 2008.

MOURA, L. G. V. Indicadores para a Avaliação da Sustentabilidade em Sistemas de Produção da Agricultura Familiar: O Caso dos Fumiculores de Agudo - RS. 2002. Dissertação (Mestrado) - Programa de Pós-Graduação em Desenvolvimento Rural. Faculdade de Ciências Econômicas. Universidade Federal do Rio Grande do Sul, Porto Alegre, 2002.
PHILIPPI JÚNIOR, A.; ROMÉRO, M. A.; BRUNA, G. C. Uma Introdução à Questão Ambiental. In: PHILIPPI JÚNIOR, A.; ROMÉRO, M. A.; BRUNA, G. C. (Ed.). Curso de Gestão Ambiental. Barueri, SP: Manole, 2004. p. 3-16.

SEPÚLVEDA, S. Desenvolvimento microregional sustentável: métodos para planejamento local. Brasília: IICA, 2005, 292 p.

SILVA, A. M.; CORREIA, A. M. M.; CÂNDIDO, G. A. Ecological Footprint Method: Avaliação da Sustentabilidade no Município de João Pessoa, PB. In: CÂNDIDO, G. A. (Org.). Desenvolvimento Sustentável e Sistemas de Indicadores de Sustentabilidade: Formas de aplicações em contextos geográficos diversos e contingências específicas. Campina Grande, PB: UFCG, 2010, p.236-271.

VAN BELLEN, H. M. Indicadores de Sustentabilidade: Uma Análise Comparativa. 2. ed. Rio de Janeiro: FGV, 2006.

VIEIRA, P. M. S.; STUDART, T. M. C. Proposta Metodológica para o Desenvolvimento de um Índice de Sustentabilidade Hidro- Ambiental de Áreas Serranas no Semiárido Brasileiro - Estudo de Caso: Maciço de Baturité, Ceará. RBRH - Revista Brasileira de Recursos Hídricos v.14, n.4, p. 125-136, out/dez, 2009.

WAQUIL, P. D.; et al. Para Medir o Desenvolvimento Territorial Rural: Validação de Uma Proposta Metodológica. In: XLV CONGRESSO DA SOCIEDADE BRASILEIRA DE ECONOMIA, ADMINISTRAÇÃO E SOCIOLOGIA RURAL. Anais... Universidade Estadual de Londrina, PR, 22 a 25 de jul. 2007, 22 p. 